\title{
Publisher Correction: Expanding the search
}

\section{Anna Dart}

Nature Reviews Cancer (2019) https://doi.org/10.1038/s41568-018-0101-9

Published online 07 January 2019

The originally published Research Highlight incorrectly credited the image. The correct credit line should read Dennis Hallinan/Alamy Stock Photo. This has now been corrected in all versions of the original article.

https://doi.org/10.1038/s41568-019-0107-y I Published online 17 January 2019 